\title{
Diez pasos para una histerectomía total por laparoscopia segura y reproducible
}

\author{
Ten steps towards a safe and feasible total laparoscopic hysterectomy
}

Juan D. Villegas-Echeverri*, Jorge D. López-Isanoa, Dany L. Piedrahita-Gutiérrez,

Claudia Bastidas-Guarín, Angélica M. Cuello-Salcedo y José D. López-Jaramillo

Unidad de Laparoscopia Ginecológica Avanzada y Dolor Pélvico, ALGIA, Clínica Comfamiliar, Pereira, Colombia

\begin{abstract}
Resumen
Objetivo: Contribuir a la formación de los especialistas describiendo de manera sistemática y pormenorizada los diez pasos para realizar una histerectomía total por laparoscopia (HTL) segura y reproducible. Método: La descripción detallada de los pasos de la HTL pretende que este procedimiento pueda ser reproducido de manera segura y efectiva. Resultados: Al conocer claramente los pasos de la HTL es posible favorecer las rutas de mínima invasión para que las pacientes se beneficien de sus múltiples beneficios demostrados. Conclusiones: Los beneficios de las rutas mínimamente invasivas para la realización de una histerectomía ya han sido demostrados en la literatura. En muy importante que los especialistas estén familiarizados con estas técnicas y que los grupos estandaricen el procedimiento para que más pacientes puedan ser intervenidas de manera segura y se beneficien de la vía laparoscópica.
\end{abstract}

Palabras clave: Histerectomía. Laparoscopia. Procedimientos quirúrgicos. Seguridad.

\begin{abstract}
Objective: To contribute to the training of specialists by describing in a systematic and detailed way the ten steps to perform a safe and feasible Total Laparoscopic Hysterectomy (HTL). Method: The detailed description of the steps of the HTL intends that this procedure can be safely and effectively reproduced. Results: By clearly knowing the steps of HTL, it is possible to favor minimally invasive routes so that patients benefit from its multiple proven benefits. Conclusions: The benefits of minimally invasive routes for performing a hysterectomy have already been demonstrated in the literature. It is particularly important that specialists are familiar with these techniques and that groups standardize the procedure so that more patients can safely undergo and benefit from the laparoscopic approach.
\end{abstract}

Key words: Hysterectomy. Laparoscopy. Surgical procedures. Safety.

\footnotetext{
Correspondencia:

*Juan D. Villegas-Echeverri

Avda. Juan B. Gutiérrez 18-60, cons. $1104 \quad$ Fecha de recepción: 03-08-2020

C.P. 660003 , Pereira, Colombia $\quad$ Fecha de aceptación: 09-12-2020

E-mail: jvillegas @ algia.com.co DOI: 10.24875/CIRU.20000845

Cir Cir. 2021;89(5):624-631

Contents available at PubMed

www.cirugiaycirujanos.com

0009-7411/@ 2020 Academia Mexicana de Cirugía. Publicado por Permanyer. Este es un artículo open access bajo la licencia CC BY-NC-ND (http://creativecommons.org/licenses/by-nc-nd/4.0/).
} 


\section{Introducción}

La histerectomía es uno de los procedimientos quirúrgicos más realizados en ginecología. El riesgo de someterse a una histerectomía durante la vida es del $20-45 \%$, dependiendo de las características sociales, económicas, culturales y tecnológicas de cada comunidad ${ }^{1-6}$

La ruta de la histerectomía benigna ha ido cambiando. La primera histerectomía vaginal planeada fue realizada por Langenbeck en 1813, pero la primera serie de casos de histerectomía vaginal fue publicada por Senn en 1895 y su tasa de mortalidad era del $75 \% \%^{7-9}$.

La primera histerectomía abdominal en la que la paciente sobrevivió fue realizada en 1853 por Burnham. La paciente se recuperó y Burnham realizó 15 histerectomías más durante los siguientes 13 años. Sin embargo, solo tres de estas pacientes sobrevivieron y las otras murieron de sepsis o hemorragia. Luego Keith implementó varias técnicas asépticas y, en 1910, la mortalidad del procedimiento disminuyó al $2.5 \%$, y así la vía abdominal se convirtió en la más utilizada, incluso hasta hoy $8,10,11$.

Los ginecólogos fuimos pioneros en la cirugía laparoscópica, y a medida que la tecnología avanzó, también lo hicieron las técnicas quirúrgicas. Harry Reich realizó la primera histerectomía total por laparoscopia (HTL) en 1989, y desde entonces cada vez más procedimientos se realizan por esta vía ${ }^{8,11,12}$.

Luego de la primera colecistectomía robótica realizada en 1997, el desarrollo llegó a las demás especialidades y en abril de 2005 la Food and Drug Administration autorizó el uso del sistema robótico $\mathrm{Da}$ Vinci (Intuitive Surgical, Inc., Sunnyvale, CA, USA) en la cirugía ginecológica ${ }^{13-16}$.

A pesar de las ventajas demostradas de la histerectomía por rutas mínimamente invasivas, todavía la vía abdominal es la más usada, sobre todo en los países de bajos y medianos ingresos. La vía laparoscópica viene creciendo de manera sostenida en los últimos años y ya en varios países es la ruta de elección $n^{13,17-20}$.

Hay diversos obstáculos para la generalización de las técnicas de mínima invasión, como dificultades para acceder al entrenamiento, curvas de aprendizaje, poca exposición de residentes a estas técnicas, barreras de los sistemas de salud, poco apoyo de los colegas que no realizan cirugía laparoscópica y percepción de un insuficiente reembolso de la cirugía mínimamente invasiva ${ }^{15,21}$.
El objetivo de este artículo es contribuir a la formación continuada de los especialistas describiendo pormenorizadamente los diez pasos para hacer una HTL de manera segura y reproducible.

Los siguientes pasos describen la manera estandarizada en que se realiza la HTL en la Unidad de Laparoscopia Ginecológica Avanzada y Dolor Pélvico, ALGIA, de la Clínica Comfamiliar, en Pereira, Colombia. ALGIA es un centro de entrenamiento en técnicas avanzadas de cirugía mínimamente invasiva y dolor pélvico. Nuestro centro realizó la primera HTL de la región hace más de 20 años y desde entonces se ha posicionado como centro de remisión, alta complejidad y con un alto volumen quirúrgico. Esperamos que el lector pueda apropiarse de este esquema quirúrgico y lo pueda aplicar en su práctica asistencial, para beneficio propio y de sus pacientes ${ }^{13,17,21}$.

El objetivo de este artículo es contribuir a la formación de los especialistas describiendo de manera sistemática y pormenorizada los diez pasos para realizar una HTL segura y reproducible.

\section{Método}

A continuación, se describen de manera pormenorizada los diez pasos usados por este grupo para hacer de la HTL un procedimiento quirúrgico seguro y reproducible. Este grupo realiza, en promedio, 250 histerectomías laparoscópicas al año. Nuestra tasa de complicaciones menores es del $3.48 \%$ y la de complicaciones mayores del $2.5 \%$. Nuestra tasa de conversión a laparotomía es del $0.23 \%$. No hemos tenido casos de mortalidad asociada con la HTL.

\section{Paso 1: preparación del procedimiento y posicionamiento de la paciente}

Todas las pacientes son vistas en la consulta prequirúrgica por uno de los integrantes de ALGIA. Es importante que las pacientes estén adecuadamente informadas de su condición, la indicación de su histerectomía, las diferentes opciones terapéuticas que tienen y los riegos y beneficios a los que se someten con el procedimiento. En la consulta ambulatoria las pacientes reciben información general de su procedimiento y los consentimientos informados que listan de manera detallada las generalidades del procedimiento y los posibles riegos de complicación, tanto de la técnica laparoscópica como de la histerectomía. 
La paciente ingresa a la sala de preparación prequirúrgica de la clínica aproximadamente 60 minutos antes de la hora programada para su procedimiento. Se confirma que la paciente está adecuadamente enterada del procedimiento, que las indicaciones preanestésicas se han cumplido y que los consentimientos informados fueron leídos y diligenciados apropiadamente. En general, no se indica preparación intestinal preoperatoria. Se aplican $2 \mathrm{~g}$ de cefazolina intravenosa como profilaxis antibiótica prequirúrgica. La paciente es trasladada a la sala de cirugía, donde uno de los cirujanos ha verificado la adecuada posición, la configuración y el funcionamiento de los equipos, incluyendo el generador electroquirúrgico, la cámara, la fuente de luz y el neumoinsuflador. La paciente es colocada en posición de litotomía dorsal antes de que se le administre la anestesia general para asegurar que no haya puntos de presión durante la cirugía. Se colocan los miembros superiores paralelos y pegados al tronco. Se asegura que no haya presión poplítea, que no haya flexión extrema de las muñecas y la cadera, y se ubican unas hombreras acolchadas para evitar el deslizamiento del cuerpo por el Trendelemburg (Fig. 1).

Se asegura la ergonomía durante el procedimiento. Por lo tanto, la mesa quirúrgica debe estar a un nivel que permita la adecuada movilidad del cirujano, y los monitores deben estar de frente al cirujano y al ayudante. Todo el equipo quirúrgico, incluyendo cirujanos, ayudantes, anestesiólogo, instrumentador quirúrgico y personal de enfermería, debe estar familiarizado con el procedimiento que se va a realizar y con los equipos utilizados. Posteriormente se verifican las listas de chequeo. Nosotros, ademas de la lista de chequeo propuesta por la Organización Mundial de la Salud, utilizamos una lista específica para laparoscopia que verifica el estado del equipo y del instrumental, y la adecuada posición de la paciente.

\section{Paso 2: sonda de Foley y manipulador uterino}

Se realiza una exploración física bajo anestesia general para confirmar los hallazgos previos y el procedimiento. Luego se procede al lavado quirúrgico abdominal y vaginal/perineal. Se inserta una sonda de Foley de $20 \mathrm{G}$ con $5 \mathrm{ml}$ en el balón antes de insertar el manipulador uterino para facilitar la colocación de este. Luego se posiciona el manipulador uterino. Nosotros utilizamos un manipulador tipo

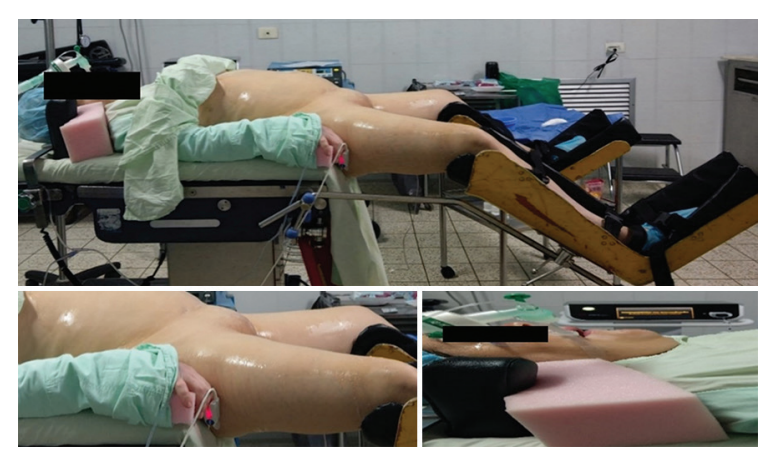

Figura 1. Posición de la paciente.

VCare ${ }^{\circledR}$ (ConMed Endosurgery, Utica, NY, USA: https://www.conmed.com/en/medical-specialties/laparoscopic-robotic-and-open-surgery/gyn -and-gyn-oncology/instruments/uterine-manipulation/ vcare-plus-and-vcare-dx), pero otros modelos, como el RUMI@ (Cooper Surgical, Trumbull, CT, USA: https://www.coopersurgical.com/medical-devices/detail/ rumi-ii-uterine-manipulator-handle) o el Clermont-Ferrand $\circledast$ (Karl Storz, Tuttlingen, Germany: https://www. karlstorz.com/cps/rde/xbcr/karlstorz_assets/ASSETS/2975530.pdf) son igual de útiles. La decisión de qué manipulador utilizar depende de la familiaridad que tenga el grupo quirúrgico con cada uno de ellos, del tamaño del cuello uterino, de la longitud y el diámetro de la vagina, del volumen uterino y del estado menopaúsico. Ocasionalmente tenemos pacientes que nunca han tenido relaciones sexuales, han estado en tratamiento prolongado con testosterona (para reasignación de sexo, por ejemplo) o tienen una marcada atrofia posmenopáusica, y en estos casos puede no utilizarse un manipulador uterino. El uso de gel de lidocaína como lubricante puede facilitar la colocación del manipulador uterino. A veces es necesario realizar una episiotomía para permitir la inserción del manipulador.

Es muy importante utilizar una copa vaginal de tamaño adecuado, que permita la delineación apropiada de los fórnices vaginales. Las copas muy grandes pueden aumentar el riesgo de lesión ureteral y las muy pequeñas no permiten delinear la vagina y separar adecuadamente la vejiga.

\section{Paso 3: ingreso abdominal y colocación de los puertos principal y accesorios}

Ninguna técnica de ingreso es más segura que las demás. Se debe usar aquella con la que el cirujano 
esté más familiarizado. Los riesgos disminuyen cuando un cirujano tiene más de 100 ingresos laparoscópicos al año con una técnica específica. Nuestro grupo utiliza la técnica de ingreso directa ${ }^{22,23}$.

El ingreso se realiza con la paciente en neutro, antes de colocarla en Trendelemburg. Usamos un trocar principal umbilical de $10 \mathrm{~mm}$ y un lente de $0^{\circ}$. Luego de la inserción del trocar, se confirma que el instrumento está intraperitoneal y se descartan lesiones relacionadas con el ingreso. Posteriormente se indica el Trendelemburg. Nosotros usamos un ángulo de $30^{\circ}$ o menor, y generalmente evitamos el Trendelemburg extremo por sus potenciales efectos deletéreos respiratorios, cardiovasculares, musculoesqueléticos y sobre el sistema nervioso central y el nervio óptico, y por el dolor posoperatorio. Se indica la creación del neumoperitoneo inicialmente a una presión de 15 $\mathrm{mmHg}^{24-27}$.

Se insertan luego tres puertos accesorios de $5 \mathrm{~mm}$ en la fosa iliaca derecha, en la fosa iliaca izquierda y en el flanco izquierdo (punto de Jain). Utilizamos una distribución que permita la sutura laparoscópica en la zona vertical, como la descrita por Koh. Los puertos (principal y accesorios) deben estar a más de $7 \mathrm{~cm}$ de distancia entre ellos para evitar el choque intraperitoneal del instrumental y facilitar la ergonomía ${ }^{28-30}$ (Fig. 2).

Es posible que los sitios de insercion de los trocares deba variar según la patología intrapélvica y el tamaño del útero, pero la distribución del puerto principal central de $10 \mathrm{~mm}$ y tres puertos accesorios de $5 \mathrm{~mm}$ generalmente se mantiene. Siempre debe tenerse en cuenta el transcurso de la vasculatura superficial y profunda de la pared abdominal, en especial de las arterias epigástricas inferiores, para evitar lesionarlas durante el ingreso de los trocares accesorios ${ }^{31-33}$.

Tan pronto como se han insertado los puertos accesorios, se disminuye la presión intraabdominal a valores entre 8 y $12 \mathrm{mmHg}$ para disminuir los eventuales efectos negativos de presiones intraabdominales de $15 \mathrm{mmHg}$ o más sobre la fisiología cardiopulmonar y el dolor posoperatorio. La adecuada visualización no se compromete con presiones bajas ${ }^{34,35}$.

En cuanto el puerto umbilical está en posición, se ha creado el neumoperitoneo, se ha dado el Trendelemburg y se han introducido los puertos accesorios, se procede a revisar la cavidad pélvica y a identificar los hallazgos intraoperatorios. Se confirman la adecuada colocación del manipulador uterino, la movilidad del útero y el acceso a los pedículos uterinos y a la copa vaginal anterior y posterior (Fig. 3).

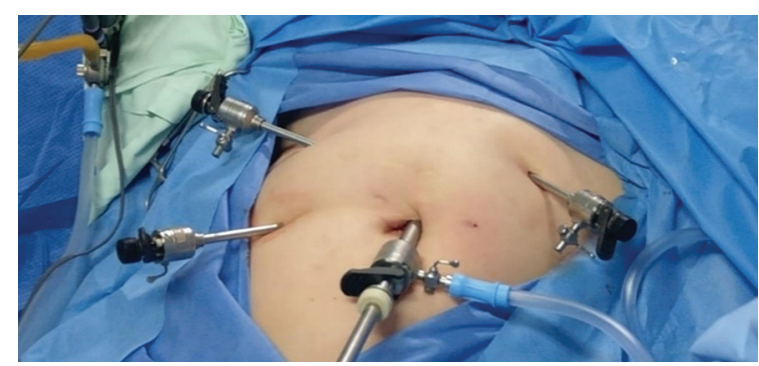

Figura 2. Posición de los trocares.

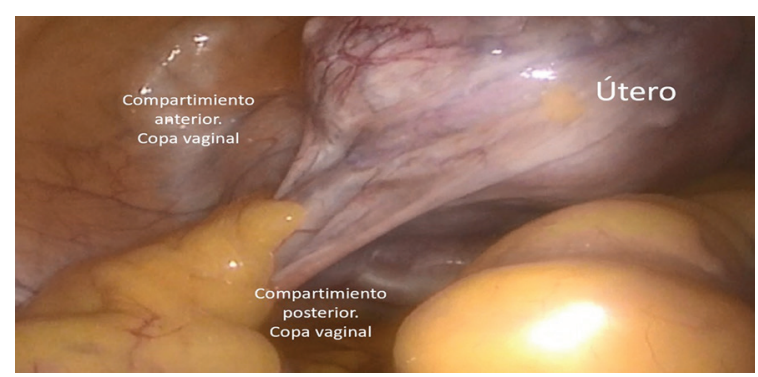

Figura 3. Posición del manipulador uterino y de la copa vaginal anterior y posterior.

\section{Paso 4: pedículos superiores}

Se realiza una salpingectomía bilateral de oportunidad en todas las pacientes y no hacemos salpingo-ooforectomía bilateral en pacientes menores de 55 años, a menos que se trate de casos especiales ${ }^{36}$.

Se procede a coagular y cortar el mesosálpinx de manera bilateral, teniendo especial cuidado con las venas que trascurren entre el ovario y el ligamento redondo, para evitar el sangrado. Luego se accede, coagulan y cortan los ligamentos útero-ováricos de manera bilateral y finalmente se hace lo mismo con ambos ligamentos redondos. La apertura del ligamento redondo proporciona acceso a los ligamentos anchos, que se separan ampliamente por tracción y contratracción. En las pacientes a quienes se les debe realizar una salpingo-ooforectomía unilateral o bilateral, primero se coagulan y seccionan los ligamentos infundíbulo pélvicos y luego los ligamentos redondos, para acceder, de la misma forma, a los ligamentos anchos (Fig. 4).

\section{Paso 5: movilización de la vejiga}

Se debe empujar firmemente el manipulador uterino para que la copa vaginal permita delinear el fórnix anterior. Se busca unir la apertura anterolateral de las 


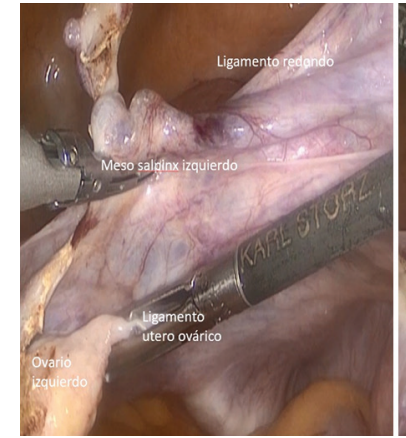

Figura 4. Pedículo superior.

hojas del ligamento ancho en la línea media. Se debe buscar el plano adecuado, donde el peritoneo se separa fácilmente con una manipulación suave. Se identifica la fascia endopélvica y se moviliza la vejiga hacia caudal. Hay que mantenerse en el tejido areolar laxo. Si se encuentra tejido graso o hay sangrado, puede ser que la disección esté demasiado cerca de la vejiga y hay que replantear los planos. En las pacientes cuya vejiga está ascendida (antecedente de cesáreas, por ejemplo) hay que mantenerse relativamente alto en el útero durante la disección (Fig. 5).

\section{Paso 6: asegurar los pedículos uterinos}

Luego de movilizar la vejiga hacia caudal, se disecan y exponen los pedículos uterinos. Hay que recordar que en los pedículos uterinos están las arterias uterinas que transcurren entre las venas uterinas anteriores superficiales y las venas uterinas profundas posteriores. Se debe empujar el manipulador uterino para alejar los vasos uterinos del uréter y delinear los fórnices vaginales anterior y posterior. Hay que asegurarse de no estar nunca por debajo de la copa vaginal para disminuir las tasas de lesión ureteral en este paso. Primero se coagula el pedículo con energía bipolar (30-35 W). Cuando las burbujas dejan de formarse, los vasos están listos para ser cortados. Luego se cortan, ya sea con tijera fría, energía ultrasónica o instrumentos de energía bipolar avanzada. El pedículo vascular debe caer hacia lateral y caudal, proporcionando un adecuado acceso avascular a la copa vaginal (Fig. 6).

\section{Paso 7: separar el útero y el cuello uterino de la cúpula vaginal}

Se identifican los fórnices vaginales mientras se presiona enérgicamente el manipulador uterino hacia
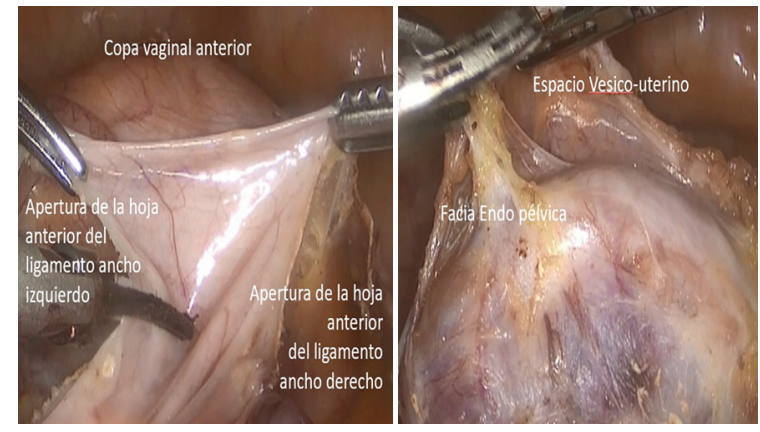

Figura 5. Espacio vésico-cérvico-vaginal.
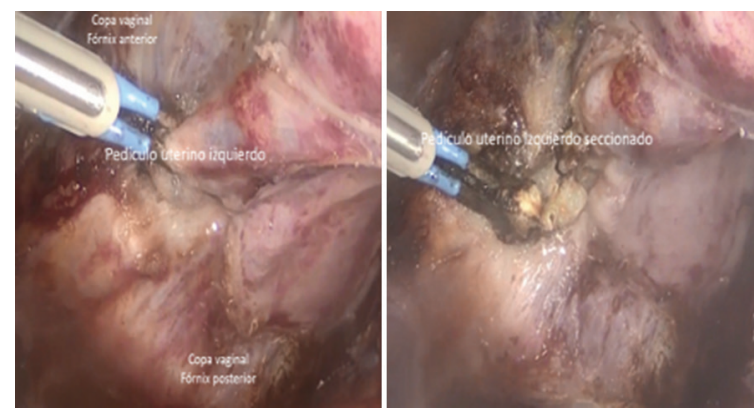

Figura 6. Pedículo inferior.

cefálico. Hay que ver y palpar la copa vaginal sobresaliendo anterior y posterior. Cuando la copa ya esté adecuadamente identificada, se procede a realizar un corte circunferencial usando un elemento de punta fina y energía monopolar (corte puro, energía no modulada) a $50 \mathrm{~W}$. Nosotros utilizamos un electrodo-gancho de corte monopolar en forma de L (Karl Storz, Tuttlingen, Germany: https://www.karlstorz.com/cps/ rde/xbcr/karlstorz_assets/ASSETS/3331472.pdf), pero existen otros disponibles en el mercado. Hay que ser cuidadoso de no cortar nunca por debajo de la copa y procurar que el corte siempre siga un sentido de posterior a anterior. También hay que asegurarse de estar cortando sobre el tejido que ya fue preparado en los pasos previos y no sobre tejido que aún no haya sido coagulado adecuadamente, para evitar demoras, pérdida de visibilidad y sangrado intraoperatorio (Fig. 7).

\section{Paso 8: remoción del útero}

Cuando finalmente el corte sobre la copa vaginal libera la pieza quirúrgica, se debe ser cuidadoso de no dejarla deslizar en la cavidad hacia cefálico y eventualmente perderla entre el contenido abdominal. El cuello 


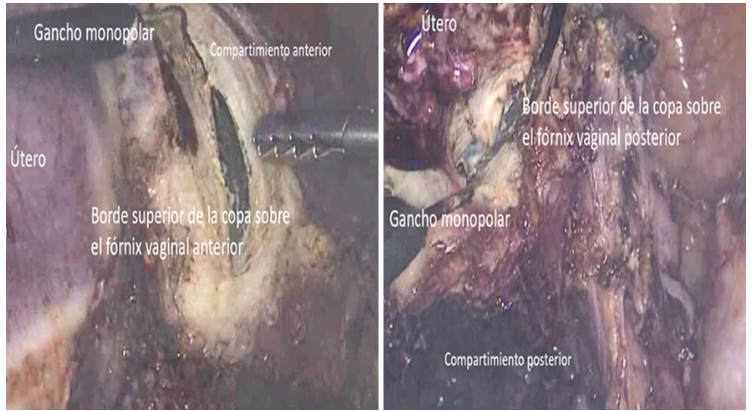

Figura 7. Apertura de la cúpula vaginal.

uterino se debe presentar en el canal vaginal y ser traccionado con una pinza tenáculo o con una pinza fuerte de triple agarre. Generalmente, el útero puede extraerse a través de la vagina sin dificultades, pero en las pacientes con úteros de gran volumen o con una vagina pequeña o poco elástica este paso puede ser desafiante. Se puede considerar la morcelación transvaginal 0 , en casos especiales, incluso el uso de un morcelador eléctrico por vía laparoscópica. Algunas veces, una episiotomía puede ayudar a la extracción. Para mantener la presión intraabdominal luego de retirar el útero, se puede ocluir el canal vaginal con unas compresas húmedas o con un guante que contenga un par de gasas en su interior. También se podría mantener el útero en el canal vaginal a manera de tapón.

\section{Paso 9: cierre de la cúpula vaginal}

Nuestro grupo siempre cierra la cúpula vaginal por vía laparoscópica, con sutura continua y en dos planos. El primer plano involucra la mucosa vaginal anterior y posterior. Comienza en el ángulo lateral derecho del manguito vaginal y continúa hacia el ángulo contralateral. El segundo plano incluye el peritoneo posterior y las fascias del anillo pericervical anterior. Se anuda donde comenzó el primer plano y así se termina el cierre de la cúpula. Generalmente utilizamos unos $22 \mathrm{~cm}$ de sutura absorbible monofilamento tipo polidioxanona 2-0 con aguja SH. No utilizamos sutura barbada, pues varios estudios han demostrado que no tiene ventajas en comparación con la otra. Además, el nuestro es un centro de formación y dos planos dan mayor exposición a la sutura al personal en entrenamiento que uno solo ${ }^{37,38}$.

Se procura realizar una suspensión de la cúpula vaginal a los ligamentos útero-sacros (McCall) en el momento del segundo plano para reducir el riesgo de prolapso apical ${ }^{39}$ (Fig. 8).

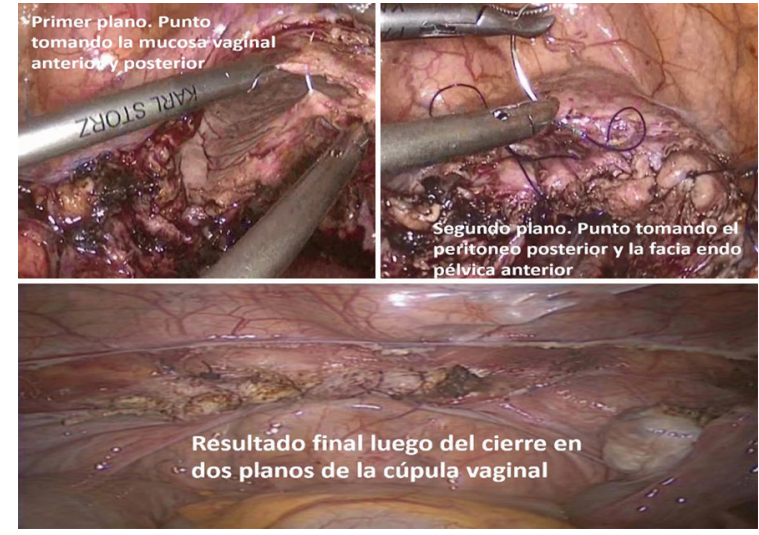

Figura 8. Cierre de la cúpula vaginal.
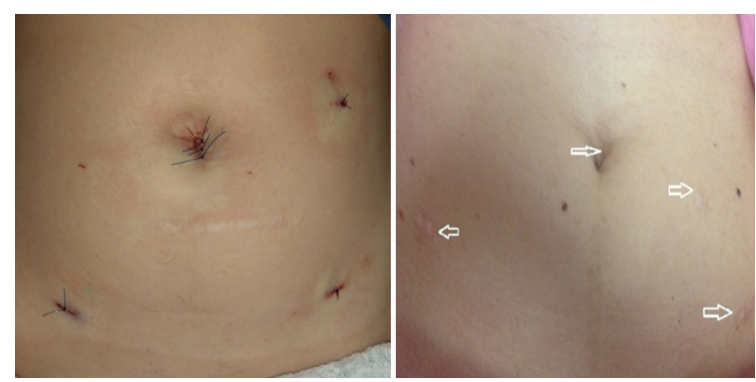

Figura 9. Sutura de la piel.

\section{Paso 10: evaluación final de la hemostasia y de la cavidad abdominopélvica. Drenaje del neumoperitoneo y cierre de los puertos y de la piel. Cuidados posoperatorios}

Finalmente se revisan cuidadosamente la hemostasia y la cavidad abdominopélvica. Se lavan y aspiran los restos de sangre y detritus, y se termina el procedimiento. Dejamos $30 \mathrm{ml}$ de bupivacaína sin epinefrina al $0.125 \%$ en la cavidad peritoneal y retiramos los trocares accesorios. Vaciamos activamente el neumoperitoneo mientras el anestesiólogo da presión positiva (maniobras de reclutamiento pulmonar), y retiramos el trocar principal. Ponemos anestésico local en las cuatro incisiones y suturamos la piel con una sutura sintética no absorbible tipo polipropileno 3-0 con aguja SC-24, que se retirará a los 7-10 días $^{40-43}$ (Fig. 9).

Al finalizar el procedimiento y luego de la extubación, la paciente es trasladada a la sala de recuperación. Es fundamental minimizar las náuseas y el vómito tras la cirugía, y se hace énfasis en el temprano y adecuado control del dolor agudo posoperatorio. 
Si la paciente evoluciona adecuadamente en las primeras 3-4 horas, es llevada a una sala de preegreso, donde se aplica el protocolo fast-track y es dada de alta luego de haber tolerado la vía oral, haberse movilizado tempranamente y de manera adecuada, y haber tenido diuresis espontánea luego del retiro de la sonda vesical, según el protocolo de recuperación mejorada después de la cirugía (ERAS, enhanced recovery after surgery). Nuestro tiempo promedio de estancia posoperatoria tras una HTL es de 13 horas $^{44-46 .}$.

La paciente egresa con recomendaciones posoperatorias estrictas, que son dadas de manera verbal y por escrito tanto a la paciente como a sus acompañantes. Se entrega fórmula médica de analgesia posquirúrgica con acetaminofeno, $1 \mathrm{~g} / 6 \mathrm{~h}$ por vía oral durante 5 días; además, un antiinflamatorio no esteroideo (diclofenaco $75 \mathrm{mg} / 12 \mathrm{~h}$ por vía intramuscular o naproxeno $250 \mathrm{mg} / 8 \mathrm{~h}$ por vía oral) durante 3 días, y metronidazol, un óvulo $(500 \mathrm{mg}$ ) intravaginal cada 12 horas durante 5 días desde el posquirúrgico inmediato. Se expide incapacidad médica por 10-14 días.

La paciente asiste a retiro de puntos y valoración por enfermería a los 7-10 días del procedimiento, y luego a control formal posoperatorio en ALGIA a los 10-14 días con el resultado de patología, y finalmente a las 6 semanas.

\section{Resultados}

Al conocer claramente los pasos de la HTL es posible favorecer las rutas de mínima invasión para que las pacientes se beneficien de sus múltiples beneficios demostrados.

\section{Discusión}

A pesar de sus beneficios reconocidos y demostrados, las vías de mínima invasión para la histerectomía siguen en desventaja al compararse con la laparotomía, sobre todo en los países de bajos y medianos ingresos. Por lo tanto, es muy importante que los grupos quirúrgicos se familiaricen y estandaricen las técnicas quirúrgicas de mínimo acceso buscando ampliar la cantidad de pacientes que se beneficien de ellas de manera segura.

\section{Conclusiones}

Los beneficios de las rutas mínimamente invasivas para la realización de una histerectomía ya han sido demostrados en la literatura. En muy importante que los especialistas estén familiarizados con estas técnicas y que los grupos estandaricen el procedimiento para que más pacientes puedan someterse de manera segura y se beneficien de la vía laparoscópica.

\section{Responsabilidades éticas}

Protección de personas y animales. Los autores declaran que para esta investigación no se han realizado experimentos en seres humanos ni en animales.

Confidencialidad de los datos. Los autores declaran que han seguido los protocolos de su centro de trabajo sobre la publicación de datos de pacientes.

Derecho a la privacidad y consentimiento informado. Los autores han obtenido el consentimiento informado de los pacientes y/o sujetos referidos en el artículo. Este documento obra en poder del autor de correspondencia.

\section{Conflicto de intereses}

Ninguno de los autores tiene conflictos de intereses que declarar con respecto a este trabajo original de investigación

\section{Bibliografía}

1. Cooper R, Hardy R, Kuh D. Timing of menarche, childbearing and hysterectomyr isk. Maturitas. 2008;61:317-22.

2. Jonsdottir GM, Jorgensen S, Cohen SL, Wright KN, Shah NT, Chavan N, et al. Increasing minimally invasive hysterectomy: effect on cost and complications. Obstet Gynecol. 2011;117:1142-9.

3. Merrill RM. Prevalence corrected hysterectomy rates and probabilities in Utah. Ann Epidemiol. 2001;11:127-35.

4. Vessey MP, Villard-Mackintosh L, McPherson K, Coulter A, Yeates D. The epidemiology of hysterectomy: findings in a large cohort study. $\mathrm{Br} J$ Obstet Gynaecol. 1992;99:402-7.

5. Wilson LF, Mishra GD. Age at menarche, level of education, parity and the risk of hysterectomy: a systematic review and meta-analyses of population-based observational studies. PLoS One. 2016;11:e0151398.

6. Stewart EA, Shuster LT, Rocca WA. Reassessing hysterectomy. Minn Med. 2012;95:36-9.

7. Byrnes JN, Occhino JA. Hysterectomy for benign conditions of the uterus: total vaginal hysterectomy. Obstet Gynecol Clin North Am. 2016;43:441-62.

8. Sutton C. Hysterectomy: a historical perspective. Baillieres Clin Obstet Gynaecol. 1997;11:1-22.

9. Moen MD, Richter HE. Vaginal hysterectomy: past, present, and future. Int Urogynecol J. 2014;25:1161-5.

10. Moen M. Hysterectomy for benign conditions of the uterus. Obstet Gynecol Clin North Am. 2016;43:431-40.

11. King CR, Giles D. Total laparoscopic hysterectomy and laparoscopic-assisted vaginal hysterectomy. Obstet Gynecol Clin North Am. 2016;43:463-78.

12. Reich H, DeCaprio J, McGlynn F. Laparoscopy hysterectomy. J Gynecol Surg. 1989;5:13-215.

13. Simpson KM, Advincula AP. The essential elements of a robotic-assisted laparoscopic hysterectomy. Obstet Gynecol Clin North Am. 2016;43: 479-93.

14. Visco A, Advincula A. Robotic gynecologic surgery. Obstet Gynecol. 2008;112:1369-84

15. Advincula A, Wang K. Evolving role and current state of robotics in minimally invasive gynecologic surgery. J Minim Invasive Gynecol. 2009;16:291-301.

16. Lawrie TA, Liu H, Lu D, Dowswell T, Song H, Wang L, et al. Robot-assisted surgery in gynaecology. Cochrane Database Syst Rev. 2019;(4):CD011. 
17. De Los Ríos JF, Castañeda JD, Calle GA, Serna E, Vásquez RA, Arango AM, et al. Histerectomía laparoscópica total en la unidad de endoscopia ginecológica de la Clínica del prado, Medellín (Colombia) 2002 - 2008. Rev. Colomb. Obstet. Ginecol. 2009;60(4):320-7.

18. Wright JD, Herzog TJ, Tsui J, Ananth CV, Lewin SN, Lu YS, et al. Nationwide trends in the performance of inpatient hysterectomy in the United States. Obstet Gynecol. 2013;122:233-41.

19. Aarts JW, Nieboer TE, Johnson N, Tavender E, Garry R, Mol BW, et al. Surgical approach to hysterectomy for benign gynaecological disease. Cochrane Database Syst Rev. 2015;(8):CD003677.

20. Turner LC, Shepherd JP, Wang L, Bunker CH, Lowder JL. Hysterectomy surgery trends: a more accurate depiction of the last decade? Am J Obstet Gynecol. 2013;208:277.e1-7.

21. Einarsson JI, Suzuki Y. Total laparoscopic hysterectomy: 10 steps toward a successful procedure. Rev Obstet Gynecol. 2009:2:57-64.

22. Ahmad G, Baker J, Finnerty J, Phillips K, Watson A. Laparoscopic entry techniques. Cochrane Database Syst Rev. 2019;(1):CD006583.

23. Vilos GA, Ternamian A, Dempster J, Laberge PY. No. 193-Laparoscopic Entry: A review of techniques, technologies, and complications. J Obstet Gynaecol Can. 2017;39:e69-e84.

24. Ghomi A, Kramer C, Askari R, Chavan NR, Einarsson JI. Trendelenburg position in gynecologic robotic-assisted surgery. J Minim Invasive Gynecol. 2012;19:485-9.

25. Gould C, Cull T, Wu YX, Osmundsen B. Blinded measure of Trendelenburg angle in pelvic robotic surgery. J Minim Invasive Gynecol. 2012;19:465-8.

26. Colombo R, Agarossi A, Borghi B, Ottolina D, Bergomi P, Ballone E, et al. The effect of prolonged steep head-down laparoscopy on the optical nerve sheath diameter. J Clin Monit Comput. 2020;34:1295-302.

27. Souki FG, Rodriguez-Blanco YF, Polu SR, Eber S, Candiotti KA. Survey of anesthesiologists' practices related to steep Trendelenburg positioning in the USA. BMC Anesthesiol. 2018;18:117.

28. Koh $\mathrm{CH}$. Laparoscopic suturing in the vertical zone. Tuttlingen: Endo Press; 2005.

29. Jain N, Jain V, Agarwal C, Bansal P, Gupta S, Bansal B. Left lateral port: safe laparoscopic port entry in previous large upper abdomen laparotomy scar. J Minim Invasive Gynecol. 2019;26:973-6.

30. Jain N, Sareen S, Kanawa S, Jain V, Gupta S, Mann S. Jain point: a new safe portal for laparoscopic entry in previous surgery cases. J Hum Reprod Sci. 2016;9:9-17.

31. Epstein J, Arora A, Ellis H. Surface anatomy of the inferior epigastric artery in relation to laparoscopic injury. Clin Anat. 2004;17:400-8.

32. Joy P, Simon B, Prithishkumar IJ, Isaac B. Topography of inferior epigastric artery relevant to laparoscopy: a CT angiographic study. Surg Radiol Anat. 2016;38:279-83.
33. Pickett SD, Rodewald KJ, Billow MR, Giannios NM, Hurd WW. Avoiding major vessel injury during laparoscopic instrument insertion. Obstet Gynecol Clin North Am. 2010;37:387-97.

34. Bogani G, Martinelli F, Ditto A, Chiappa V, Lorusso D, Ghezzi F, et al. Pneumoperitoneum pressures during pelvic laparoscopic surgery: a systematic review and meta-analysis. Eur J Obstet Gynecol Reprod Biol. 2015;195:1-6.

35. Topçu HO, Cavkaytar S, Kokanalı K, Guzel Al, Islimye M, Doganay M. A prospective randomized trial of postoperative pain following different insufflation pressures during gynecologic laparoscopy. Eur J Obstet Gynecol Reprod Biol. 2014;182:81-5.

36. ACOG Committee Opinion No. 774: Opportunistic salpingectomy as a strategy for epithelial ovarian cancer prevention. Systematic opportunity bilateral salpingectomy. Obstet Gynecol. 2019;133:e279-84.

37. López CC, Ríos JFL, González Y, Vásquez-Trespalacios EM, Serna D, Arango $\mathrm{A}$, et al. Barbed suture versus conventional suture for vaginal cuff closure in total laparoscopic hysterectomy: randomized controlled clinical trial. J Minim Invasive Gynecol. 2019;26:1104-9.

38. Yildirim D, Ozyurek SE, Kiyak H, Han A, Koroglu N, Bestel A, et al. Single-layer versus double-layer closure of the vaginal cuff with barbed sutures in laparoscopic hysterectomy. Ginekol Pol. 2018;89:229-34.

39. Hodgson B. AAGL practice report: Practice guidelines on the prevention of apical prolapse at the time of benign hysterectomy. J Minim Invasive Gynecol. 2014;21:715-22.

40. Kaloo P, Armstrong S, Kaloo C, Jordan V. Interventions to reduce shoulder pain following gynaecological laparoscopic procedures. Cochrane Database Syst Rev. 2019;(1):CD011101.

41. Lee J, Park C, Kim J, Ki Y, Cha SH, Kim JY. Effect of low-pressure pulmonary recruitment maneuver on postlaparoscopic shoulder pain: randomized controlled trial. J Minim Invasive Gynecol. 2020;27:173-7.

42. Ravndal C, Vandrevala T. Preemptive local anesthetic in gynecologic laparoscopy and postoperative movement-evoked pain: a randomized trial. J Minim Invasive Gynecol. 2016;23:775-80.

43. Blanton E, Lamvu G, Patanwala I, Barron KI, Witzeman K, Tu FF, et al. Non-opioid pain management in benign minimally invasive hysterectomy: a systematic review. Am J Obstet Gynecol. 2017;216:557-67.

44. Committee on Gynecologic Practice. ACOG Committee Opinion No. 750: Perioperative pathways: enhanced recovery after surgery. Obstet Gynecol. 2018;132:e120-30.

45. Carey ET, Moulder JK. Perioperative management and implementation of enhanced recovery programs in gynecologic surgery for benign indications. Obstet Gynecol. 2018;132:137-46.

46. Kalogera E, Dowdy SC. Enhanced recovery pathway in gynecologic surgery: improving outcomes through evidence-based medicine. Obstet Gynecol Clin North Am. 2016;43:551-73. 